\title{
Forecasting about EURJPY exchange rate using hidden Markova model and CART classification algorithm
}

\author{
Abdorrahman Haeri ${ }^{1,2}$ *, Seyed Morteza Hatefi ${ }^{3}$, Kamran Rezaie ${ }^{4}$ \\ ${ }^{1}$ School of Industrial Engineering, Iran University of Science \& Technology, Tehran, Iran \\ ${ }^{2}$ Center of excellence for optimization of production and service systems, Iran University of Science \& Technology, Tehran, Iran \\ ${ }^{3}$ Faculty of Engineering, Shahrekord University, Rahbar Boulevard, P.O. Box 115, Shahrekord, Iran \\ ${ }^{4}$ School of Industrial Engineering, College of Engineering, University of Tehran, Tehran, Iran \\ *Corresponding author E-mail: ahaeri@iust.ac.ir
}

Copyright () 2015 Abdorrahman Haeri et al. This is an open access article distributed under the Creative Commons Attribution License, which permits unrestricted use, distribution, and reproduction in any medium, provided the original work is properly cited.

\begin{abstract}
The goal of this paper is forecasting direction (increase or decrease) of EURJPY exchange rate in a day. For this purpose five major indicators are used. The indicators are exponential moving average (EMA), stochastic oscillator (KD), moving average convergence divergence (MACD), relative strength index (RSI) and Williams \% (WMS \% R). Then a hybrid approach using hidden Markov models and CART classification algorithms is developed. Proposed approach is used for forecasting direcation (increase or decrease) of Euro-Yen exchange rates in a day. Also the approach is used for forecasting differnece between intial and maximum exchange rates in a day. As well as it is used for forecasting differnece between intial and minimum exchange rates in a day. Reslut of proposed method is compared with CART and neural network. Comparison shows that the forecasting with proposed method has higher accuracy.
\end{abstract}

Keywords: CART Classification Algorithm; EURJPY Exchange Rate; Forecasting; Foreign Exchange Market; Hidden Markov Model; Indicators; Neural Network.

\section{Introduction}

Initially an overview of foreign exchange market (Forex) is presented. In Forex market, the traders exchange different currencies via Internet. There are three major orders for trading.

- Buy order: Consider that analysis expresses that the EURJPY exchange rate will increase soon. In this condition, the trader request "Buy" order.

- Sell order: Consider that analysis expresses that the EURJPY exchange rate will decrease soon. In this conditions, the trader request "Sell" order.

- Close order: Each opened trade must be closed at a time. The suitable time for trade closing depends to trader analysis. For example closing can be occurred when the trader is acquiescent for trade profit. As well as, closing can be occurred when the trader is afraid of the trade loss.

Moreover traders can assign two limits to their trades. These limits are called stop loss (SL) and take profit (TP). After trade opening if the loss of that trade reaches to a specific limit (SL), the broker automatically close that trade. Also if the profit of a trade reaches to a specific limit (TP), the broker automatically close that trade. These limits are used for decreasing trade risks.

There are four major exchange rates in each time period. A time period is a time interval such as an hour, a day, a week and etc.

- $\quad$ Exchange rate at the start of a period. This exchange rate is called Open.

- Maximum value of exchange rate during a period. This exchange rate is called high.

- $\quad$ Minimum value of exchange rate during a period. This exchange rate is called Low.

- $\quad$ Exchange rate at the end of a period. This exchange rate is called Close. 
In Forex market, usually profit, loss and exchange rate changes are stated on the basis of the Pip. Pip is a criterion for exchange rate changes. For example consider that EURJPY exchange rate changes from 158.75 to 158.76 . In this condition it is said that EURJPY exchange rate increases one pip. If EURUSD exchange rate changes from 1.4595 to 1.4594, it is said that EURUSD exchange rate decreases one pip. At whole one pip is equal to one unit change in the last digit of exchange rate.

There are two general approaches for analysis and forecasting in Forex market. The approaches are technical and fundamental. In technical methods, analysis and forecasting are performed on the basis of the exchange rate and technical indicators such as moving average, RSI and etc. In fundamental methods, analysis and forecasting are performed on the basis of the immense variables such as interest rate, unemployment rate, GDP and etc. On the basis of these investigations forecasting about increase or decrease of a currency versus other currencies is presented. Political events and immense decisions has affected on the fundamental analysis.

\section{Literature review}

In this section a review about some of the previous researches about exchange rate forecasting and decision making in foreign exchange market is presented. By the attention to paper approach, reviewed papers are about the technical approach.

Chew Lim Tan and J. Yao [1] develop a neural network for forecasting in Forex market. Time series and moving average indicator are the inputs of back propagation neural network. They presented forecasting about Japanese yen, British pound, Switzerland Frank and Australian dollar versus US dollar. A.Muriel [2] used of a chaotic moving model in physics. He tried to forecast trend of three major currencies versus US dollar. The model is tested on October 2003 date set. Fang-Mei Tseng et. al. [3] combined ARIMA (Auto Regressign Integrated Moving Average) in time seris and fuzzy regression model to forecasting NT (New Tawanian) dollar versus US dollar. The model enables decision makers and traders to forecast the worst and the best status in future. Chakradhara Panda and V.Narasimhan [4] used of feed forward neural network to forecast Indian Rupee versus US dollar weekly exchange rate. Results show that proposed model is better than linear regression and random walk models. Vincent C.S Lee and Hsiao Tshung Wong [5] used neural network to develop a risk management model. Aim of the model was forecasting direction and value of exchange rate changes. Proposed model was a combined model and used of ANN (Artificial neural network) and fuzzy logic tools. Roberto Baviera [6] investigated non-reliable Markovian behavior of exchange rate. He used from that to inference special rules for doing successful trades. Craig Ellis and Patrick Wilson [7] developed an integrated approach for forecasting foreign exchange rate. They used of random walk framework to forecasting direction and value of exchange rates. They estimated different statistical criteria such as mean, standard deviation, p-value and etc. Thomas ET. al. [8] examined random behavior hypothesis in foreign exchange market. The focus of this research is on the future contracts. They used of regression for detecting relations among two consecutive periods. Data set includes exchange rate of Canadian dollar, Australian dollar, Japanese yen, Switzerland frank, British pound and Euro versus US dollar. At the end a profitable strategy was presented. Gan ET. al. [9] used multi layered perceptron (MLP) neural network to forecasting exchange rate of Switzerland frank, Deutsche Mark and Japanese yen versus US dollar. They examined two models with single and multivariate time series and compared them with random walk model. Oh, K.J. ET. al. [10] by using of nonlinear programming and neural network developed a daily financial condition indicator (DFCI) for presenting time signals. Their Proposed indicator had capability to create a zone alarm region for forecasting stochastic financial crisis. At the end, he used of DFCI on Korean financial market. Hau, H. et al. [11] developed a balanced model among foreign exchange rate, price stock and capital flow. Their results show that net flow in foreign exchange market has a positive correlation with value of currency unit. Their Results show that forecasting on the daily, monthly and seasonal time horizons have acceptable confidence. Ahmad, S.M. et al. [12] by combining the famous technical indicators, developed a fuzzy indicator for presenting buy and sell signals in Forex and stock market. Results of their research show that proposed indicator had higher confidence than other indicators. Mei-Chih-Chen et. al. [13] by using of moving average (MA), stochastic indicators (KD), moving average convergence divergence (MACD), relative strength index (RSI) and Williams \%R (WMS \%R) developed a dynamic stock portfolio decision-making assistance model to forecasting direction of price changes in Taiwan stock market. Thomas C. Shik. ET. al. [14] compares RSI and moving average on six currencies. Terence Tai-Leung Chong and Wing-Kam Ng [15] investigated the beneficiary efficacy of rules that extracted from RSI and MACD in London stock market.

\section{Problem definition}

In this paper a hybrid approach from hidden Markov model (HMM) and CART classification algorithm is used for forecasting about euro-yen (That is called EURJPY) exchange rate. Initially direction (increase or decrease) of EURJPY exchange rate in day is considered. Then the approach is used for forecasting differnece between High and Open exchange rate in day. Alos the approach is used for forecasting differnece between Open and Low exchange rate in day. At the end results of proposed approach are compared with results of CART classification algorithm and neural network. 
There are many indicators for analysis and decision making in Forex market. Traders use indicators and exchange rate changes for analysis. In this paper, five major indicators on the basis of the [13] are used for forecasting. The indicators are exponential moving average (EMA), stochastic indicators (KD), moving average convergence divergence (MACD), relative strength index (RSI) and Williams \% R (WMS \% R). A short description about the indicators are mentioned as follows:

- $\quad$ Exponential moving average (EMA): EMA is weighted average of exchange rate at previous days. EMA is a useful indicator that assigns grater weights to the latest data to respond faster to changes.

- Stochastic oscillator (KD): Stochastic oscillator is based on as exchange rate is advanced, closing exchange rate tend to move to upper of its range. This indicator uses two moving averages and converts them to two \% $\mathrm{K}$ and $\% \mathrm{D}$ lines that swing permanently between 0 and 100 . So this indicator also called KD. When value of KD is more than 80, it is a signal that exchange rate is placed on the upper bound. Also when value of KD is less than 20, it is a signal that exchange rate is placed on the lower bound.

- $\quad$ Moving average convergence divergence (MACD): Moving average convergence divergence (MACD) is equal to difference between two moving averages. One moving average has smaller length such as 12 and called fast EMA. The other moving average has larger length such as 26 and called slow EMA

- $\quad$ Relative strength index (RSI): RSI is a useful and practical tool for showing exchange rates swings. RSI compares increase value of exchange rate or stock price by decrease value over a specific period. RSI converts that information to a number between 0 and 100 .

- Williams \% (WMS \% R): William percent range technical indicator is an indicator to recognizing upper and lower limits of exchange rate. This is similar to stochastic oscillator.

\subsection{Description about hidden Markova models (HMM)}

A Markov chain is a sequence of stochastic variables. The state of each stage in a Markov chain depends only to the state of previous stage. Hidden Markov chain is one kind of the Markov chains. States of a hidden Markov chains divide to observable states and hidden states. Observable states are the states that their conditions are clear. Hidden states are the states that their conditions are not clear. For example consider a two person game. One person has a biased and an unbiased die. In each stage he throws one of the dies. For other person the face of thrown die is an observable state. But the kind of thrown die is hidden. The goal is attaining the probability of each hidden state occurring. For reaching to this goal hidden Markov models use observable stets. Indeed for each case hidden Markov models try to calculate probability of occurrence of each hidden state using of condition of observable states. For example consider that there are two hidden states. The probability of one of hidden state is $\alpha$. So the probability of the other hidden state is equal to 1- $\alpha$. A is calculated on the basis of (1) and (2) as follows:

$\tau=\frac{\sum_{j=1}^{n}\left(q_{j}-p_{2 j}\right)\left(p_{1 j}-p_{2 j}\right)}{\sum_{j=1}^{n}\left(p_{1 j}-p_{2 j}\right)^{2}}$

$\alpha=\left\{\begin{array}{lll}0 & \text { if } & \tau \leq 0 \\ \tau & \text { if } & 0<\tau<1 \\ 1 & \text { if } & \tau \geq 1\end{array}\right\}$

Consider that $\mathrm{j}$ indicates $\mathrm{j}$-th hidden state. $\mathrm{P} 1 \mathrm{j}$ is the average value of observable state $\mathrm{j}$ when the first hidden state is occurred. As well as $p_{2 j}$ is the average value of observable state $j$ when the second hidden state is occurred. Note that in this paper stages are stated as days. So at the beginning of each day the average value of previous days is specific. So $\mathrm{p}_{1 \mathrm{j}}$ is the average value of observable state $\mathrm{j}$ in $(\mathrm{j}-1)$ previous days when the first hidden state is occurred. Also $\mathrm{p}_{2 \mathrm{j}}$ is the average value of observable state $\mathrm{j}$ in $(\mathrm{j}-1)$ previous days when the second hidden state is occurred. $\mathrm{q}_{\mathrm{j}}$ is the average value of observable state j. $\tau$ Is calculated from equation (1). Then $\alpha$ is attained from equation (2). After achieving $\alpha$, it is necessary to present a decision making criterion to forecast about hidden states. For this purpose CART, a major and strong classification algorithm, is used. Indeed CART tries to find the best relationship among values of $\alpha$ and hidden states. So the proposed model first attains $\alpha$ value on the basis of the observable states. Then on the basis of the value of $\alpha$ and using of CART algorithm, decides to present forecasting about hidden states. So this method is a combined approach from HMM model and CART algorithm.

It is possible to consider observable and hidden states in financial markets such as Forex. At whole it can be said that all of history data are observable states. Also all of data that are belongs to future are hidden states. In this paper, values of five indicators (EMA, KD, MACD, RSI and WMS) at the beginning of each day are considered to define observable states. For each indicator two fields are defined. One Field includes normalized value of indicator. Max-min method is 
used for normalization. For example normalized value of moving average indicator in day (i) is calculated from equation 3 .

$$
M A(N)_{i}=\frac{\operatorname{MA}(N)_{i}-\min \sum_{i=1}^{i=n} M A(N)_{i}}{\max \sum_{i=1}^{i=n} M A(N)_{i}-\min \sum_{i=1}^{i=n} M A(N)_{i}}
$$

In above relation, $\mathrm{n}$ is number of total of days that is considered. The other Field equals to change percentage of each normalized value of an indicator. For example, change percentage of moving average indicator in period i is calculated from equation (4).

$$
M A(P)_{i}=\frac{M A(N)_{i}-M A(N)_{i-1}}{M A(N)_{i-1}}
$$

So there are ten fields for forecasting. The fields are: $\mathrm{MA}(\mathrm{N}), \mathrm{KD}(\mathrm{N}), \mathrm{MACD}(\mathrm{N}), \mathrm{RSI}(\mathrm{N}), \mathrm{WMS}(\mathrm{N}), \mathrm{MA}(\mathrm{P}), \mathrm{KD}(\mathrm{P})$, $\operatorname{MACD}(\mathrm{P}), \mathrm{RSI}(\mathrm{P})$ and WMS(P). Values of those ten fields are observable states in whole of this paper. This procedure for defining observable states is similar to [13].

\subsection{Forecasting about direction of exchange rate}

Enough descriptions about observable states are presented in previous section. Now it is necessary to define hidden states. Hidden states relate to goal of forecasting. In this section direction of exchange rate changes in a day is considered as hidden state and is showed with D. If exchange rate at the end of a day (Close) is greater than exchange rate at the start of that day (Open), then the value of hidden state is equal to 1 . Also if exchange rate at the end of a day (Close) is less than or equal to exchange rate at the start of that day (Open), then the value of hidden state is equal to 0.

\subsection{Forecasting about the value of exchange rate change}

In previous section the direction of exchange rate changes is considered to hidden state. In this section the measure of exchange rate changes is considered as hidden state. It is said in section 1 that there are four important exchange rates (Open, High, Low and close) in a day. Traders in each day attend to these four exchange rates. Differences among said rates such as (High-Open) (that is called HO) and (Open-Low) (than is called OL) are important for traders and investors. Indeed Each trader is interested to answer to these questions: At least how much exchange rate increase in a day? Or at least how much exchange rate decrease in a day? Answers of these questions are very important. For example consider that analysis is stated that in future day exchange rate increase 30 pips. So trader can open a buy trade at the beginning of a day and sets take profit (TP) of the trade equal to the point that exchange rate increase 30 pips. As well as consider that analysis is stated that in future day exchange rate decrease 20 pips. So trader can open a sell trade at the beginning of a day and sets take profit of the trade equal to the point that exchange rate decrease 20 pips.

For achieving to answer of the said questions two new variables are defined. The variables are HON and OLN. Values of those variables are attained as follows:

$$
\begin{array}{lll}
\text { If } \mathrm{HO} \geq \mathrm{A} \text { then } \mathrm{HON}=1 & \text { else } & \mathrm{HON}=0 \\
\text { If } \mathrm{OL} \geq \mathrm{A} \text { then } \mathrm{OLN}=1 & \text { else } & \mathrm{OLN}=0
\end{array}
$$

$\mathrm{A}$ is a constant value that can be used for adjusting take profit (TP) values. Relation (5) ensures that if difference among High and Open exchange rates is greater than or equal to A, then a binary variable (that is called HON) is equal to 1. Otherwise HON is equal to 0. Also Relation (6) ensures that if difference among Open and Low exchange rates are greater than or equal to A, then a binary variable (that is called OLN) is equal to 1 . Otherwise OLN is equal to 0 . Note that HO, OL and A is stated on the basis of the Pip. In this case values of HON and OLN are hidden states and are forecasted on the basis of proposed approach.

\section{Computational results and discussion}

In this section results of the proposed approach on training and testing data set are shown. EURJPY daily exchange rates in 2002 to 2007 years are used for training. After that, EURJPY daily exchange rates in the first six months of 2008 are used for testing. Also results of the proposed model are compared with CART algorithm and neural networks. 
CART needs some input variables and a target variable. The values of MA(N), KD(N), MACD(N), RSI(N), WMS(N), $\mathrm{MA}(\mathrm{P}), \mathrm{KD}(\mathrm{P}), \mathrm{MACD}(\mathrm{P}), \mathrm{RSI}(\mathrm{P})$ and $\mathrm{WMS}(\mathrm{P})$ are the input variables. Value of hidden state in each case is target variable. So $\mathrm{D}, \mathrm{HON}$ and OLN are target variables.

Neural networks consist of one input layer, number of hidden layers and one output layer. Structure of neural network is similar to used networks in [4]. So a feed-forward algorithm with a single hidden layer with 20 nodes is used. Transfer function is sigmoid or logistic. Values of MA(N), KD(N), MACD(N), RSI(N), WMS(N), MA(P), KD(P), MACD(P), $\mathrm{RSI}(\mathrm{P})$ and WMS(P) constitute input layer. Values of D, HO and OL (separately) form output layer.

Table 1 shows results of three approaches for forecasting direction of daily exchange rate (D variable).

Table 1: Accuracy of Three Methods for Forecasting the Direction

\begin{tabular}{llll}
\hline & CART & NN & Proposed method \\
\hline Accuracy on training data set & $59.29 \%$ & $55.06 \%$ & $55.64 \%$ \\
Accuracy on testing data set & $49.22 \%$ & $53.91 \%$ & $53.91 \%$ \\
\hline
\end{tabular}

Table 1 shows that accuracy of the proposed method is greater than or equal to accuracy of the other methods on testing data set.

Table 2 shows results of three approaches for forecasting about the difference among High and Open (HON variable). Calculations are performed for five values for A.

Table 2: Accuracy of Three Methods for Forecasting HON Variable

\begin{tabular}{lllllll}
\hline & \multicolumn{3}{l}{ Accuracy on training data set } & \multicolumn{3}{l}{ Accuracy on testing data set } \\
A & CART & NN & Proposed method & CART & NN & Proposed method \\
\hline 10 & 91.15 & 91.15 & 91.15 & 89.84 & 89.84 & 89.84 \\
20 & 80.26 & 79.81 & 79.87 & 82.03 & 82.03 & 82.03 \\
30 & 68.14 & 68.14 & 68.14 & 72.66 & 72.66 & 72.66 \\
40 & 59.29 & 46.15 & 57.95 & 58.59 & 42.19 & 66.41 \\
50 & 60.58 & 53.46 & 54.74 & 46.09 & 39.06 & 47.66 \\
Average & 71.884 & 67.74 & 70.37 & 69.84 & 65.16 & 71.72 \\
\hline
\end{tabular}

Table 2 shows that the accuracy of the proposed method for all value of $\mathrm{A}$ is greater than the other methods on testing data set.

Table 3 shows results of three approaches for forecasting about the difference among Open and Low (OLN variable). Calculations are performed for five values for A.

Table 3: Accuracy of Three Methods for Forecasting OLN Variable

\begin{tabular}{lllllll}
\hline & \multicolumn{2}{l}{ Accuracy on training data set } & \multicolumn{3}{l}{ Accuracy on testing data set } \\
A & CART & NN & Proposed method & CART & NN & Proposed method \\
\hline 10 & 90.64 & 90.38 & 90.38 & 86.72 & 89.06 & 89.06 \\
20 & 78.27 & 77.5 & 77.5 & 83.59 & 82.81 & 82.81 \\
30 & 66.86 & 65.51 & 65.51 & 65.62 & 67.97 & 67.97 \\
40 & 61.54 & 54.1 & 53.4 & 51.56 & 56.25 & 64.84 \\
50 & 61.03 & 56.54 & 56.47 & 49.22 & 46.88 & 41.41 \\
Average & 71.668 & 68.806 & 68.652 & 67.342 & 68.594 & 69.218 \\
\hline
\end{tabular}

Table 3 shows that the accuracy of the proposed method for all value of A except 20 and 50 is greater than the other methods on testing data set. Also table 3 shows that in average the accuracy of proposed model is greater than the others on testing data set.

\section{Conclusion $\&$ future research}

In this paper a proposed approach for forecasting in foreign exchange market is presented. Five major indicators (EMA, KD, MACD, RSI and WMS) are used for forecasting. The proposed approach uses hidden Markov model and CART classification algorithm. Three goals for forecasting are considered. First direction of exchange rate in a day (increase or decrease) is forecasted. Then forecasting about difference among High and Open exchange rates is performed. Also forecasting about difference among Open and Low exchange rates is considered. Data set is divided to two sections. First section includes EURJPY daily exchange rates in 2002 to 2007 years and used for training. Second section includes EURJPY daily exchange rates in the first six months of 2008 are used for testing. Results of the proposed model are compared with CART algorithm and neural network approach. Results show that accuracy of the proposed method is better than the accuracy of the other two methods. The novelties of this paper are:

- Using efficiently of hidden Markov model to forecast in foreign exchange market

- $\quad$ Present forecasting about difference among High and Open exchange rates and Open and Low exchange rates 
- Using a classification algorithm for forecasting

- $\quad$ Considering five major indicators for forecasting

At the end an offer for future research is presented. It is possible to present profitable trading strategy on the basis of the considered models that are used for forecasting. That can be a good topic for a future research. Also researches can use other forecasting methods on the outputs of proposed method to increase the accuracy of forecasting.

\section{References}

[1] Jingtao Yao and Chew Lim Tan, "A case study on using neural networks to perform technical forecasting of Forex" Neurocomputing, Vol. 34, No. 1-4, (2000), pp. 79-98. http://dx.doi.org/10.1016/S0925-2312(00)00300-3.

[2] A. Muriel, "Short-term predictions in Forex trading", Physica A: Statistical Mechanics and its Applications, Vol. 344, No. 1-2, (2004), pp. 190-193.

[3] Fang-Mei Tseng, Gwo-Hshiung Tzeng, Hsiao-Cheng Yu and Benjamin J. C. Yuan, "Fuzzy ARIMA model for forecasting the foreign exchange market", Fuzzy Sets and Systems, Vol. 118, No. 1, (2001), pp. 9-19. http://dx.doi.org/10.1016/S0165-0114(98)00286-3.

[4] Chakradhara Panda and V. Narasimhan, "Forecasting exchange rate better with artificial neural network", Journal of Policy Modeling, Vol. 29, No. 2, (2007), pp. 227-236. http://dx.doi.org/10.1016/j.jpolmod.2006.01.005.

[5] Vincent C.S. Lee and Hsiao Tshung Wong, "A multivariate neuro-fuzzy system for foreign currency risk management decision making", Neurocomputing, Vol. 70, No. 4-6, (2007), pp. 942-951. http://dx.doi.org/10.1016/j.neucom.2006.10.025.

[6] Roberto Baviera, Michele Pasquini, Maurizio Serva, Davide Vergni and Angelo Vulpiani, "Antipersistent Markov behavior in foreign exchange markets", Physica A: Statistical Mechanics and its Applications, Vol. 312, No. 3-4, (2002), pp. 565-576.

[7] Craig Ellis, Patrick Wilson, "A stochastic approach to modeling the USD/AUD exchange rate", International Journal of Managerial Finance,Vol. 1,No. 1, (2005), pp.36-48. http://dx.doi.org/10.1108/17439130510584874.

[8] Kuntara Pukthuanthong,Lee R. Thomas III,Carlos Bazan, "Random walk currency futures profits revisited", International Journal of Managerial Finance, Vol. 3, No.3, (2007), pp. 263-286. http://dx.doi.org/10.1108/17439130710756916.

[9] Gan, Woon-Seng, Ng, Kah-Hwa, "Multivariate Forex forecasting using artificial neural networks", Proceedings of the IEEE International Conference on Neural Networks, Vol. 2, (1995), pp: 1018-1022. http://dx.doi.org/10.1109/ICNN.1995.487560.

[10] Oh, K.J., Kim, T.Y., Lee, H.Y., Lee, H., "Using neural networks to support early warning system for financial crisis forecasting", Lecture Notes in Computer Science (including subseries Lecture Notes in Artificial Intelligence and Lecture Notes in Bioinformatics), Vol. 3809, (2005), pp. 284-296.

[11] Hau, H., Rey, H., "Exchange rates, equity prices, and capital flows", Review of Financial Studies, Vol. 19, No. 1, (2006), pp. $273-317$. http://dx.doi.org/10.1093/rfs/hhj008.

[12] Ahmad, S.M., El Gayar, N., Abd Elazim, H.Y., "A fuzzy engine model for financial market prediction", WSEAS Transactions on Information Science and Applications, Vol. 4, No. 2, (2007), pp. 362-368.

[13] Mei-Chih Chen · Chang-Li Lin · An-Pin Chen, "Constructing a dynamic stock portfolio decision-making assistance model: using the Taiwan 50 Index constituents as an example", Soft Computing, Vol. 11, (2007), pp. 1149-1156. http://dx.doi.org/10.1007/s00500-007-0158-y.

[14] Shik, T.C., Chong, T.T.-L., "A comparison of MA and RSI returns with exchange rate intervention", Applied Economics Letters, Vol. 14, No. 5, (2007), pp. 371-383. http://dx.doi.org/10.1080/13504850500426269.

[15] Chong, T.T.-L., Ng, W.-K., "Technical analysis and the London stock exchange: Testing the MACD and RSI rules using the FT30", Applied Economics Letters, Vol. 15, No. 14, (2008), pp. 1111-1114. http://dx.doi.org/10.1080/13504850600993598. 